\title{
A Modified Approach for Drawing Force Estimation of Bi-metallic Composite Pipe Forming
}

\author{
M. Zheng ${ }^{1, a}$, J. $\mathrm{Yu}^{2, \mathrm{~b}}$ \\ ${ }^{1}$ School of Chemical Engineering, Northwest University, Xián, 710069, China \\ ${ }^{2}$ School of Life Science, Northwest University, Xián, 710069, China \\ amszheng2@yahoo.com, byujie@nwu.edu.cn
}

Keywords: bi-metallic pipe, composite, forming, drawing force, residual stress.

\begin{abstract}
In this article, a modified approach for drawing force estimation of bi-metallic composite pipe forming is proposed in the light of the results of FEM simulation, the assumptions of plane strain and elastic-perfectly plastic material model are employed additionally. The modified approach for drawing force estimation of bi-metallic composite pipe forming contains the fundamental material properties and geometric parameters of both liner pipe and the base pipe.
\end{abstract}

\section{Introduction}

In recent years, the widely used transportation approach is pipeline in petroleum, chemical engineering, metallurgy, electric power and city gas, heating and water supply systems. The safety of the pipeline is a very important problem due to the attendant corrosions, such problem is growing tremendously with the increasing use of pipeline.

Bi-metallic composite pipe is a new type of pipeline, it has been proposed to solve the corrosion problem these years. It consists of two different metal pipes, i.e., one kind of inner pipe (liner pipe), another outer pipe (base pipe). Both pipes are connected closely through deformation or other technique. In general, the base pipe is to withstand for the requirement of stress and the inner lining pipe material is to meet the demands of corrosion or abrasion in such bimetallic composite pipe. As a result, the composite pipe has the comprehensive advantages of both the base pipe and the liner pipe materials. It has not only high strength, but also excellent anti-corrosion, wear resistance and other properties. Therefore, the bi-metallic composite pipe has a bright future in petroleum, chemical engineering, nuclear industry, medical, food, construction, fire and other fields.

In general, the production of bi-metal composite pipe can be divided into plastic forming mode and non-plastic forming mode. In the plastic pipe forming technology, the pipe is plastic deformed locally or totally to perform the forming process of the inner and the outer pipes; In recent years, the plastic forming mode is widely used at home and abroad since the plastic pipe forming technology has advantage of high efficiency with simple process and equipment. The plastic forming mode includes mainly mechanical drawing method, rolling method, hydro-forming method and explosive forming method.

The mechanical drawing and hydro-forming methods are the important processes of bi-metallic composite pipe production. Therefore, it is important to analyze the deformation and stress conditions in the forming process of bi-metallic composite pipe by mechanical drawing and hydro-forming methods, so that rational and effective design of the production process could be conducted.

Generally, rather complex plastic deforming process is involved in mechanical drawing and hydraulic bulging processes for bi-metallic composite pipe manufacturing. Both physical and geometric nonlinearity is contained, in addition to the complexity of the boundary conditions [1-3], which make it difficult to establish a rigorous theoretical analysis for drawing forming and hydraulic bulging of bi-metallic composite pipe. Some researchers employed finite element simulation to perform relevant analysis, good results were obtained [4-7]; In 1984, Masashi Takemoto gave the relationship between the expanding pressure $P_{i}$ and the contact pressure $P_{c}$ by assuming Tresca yield 
criterion and ideal elastic-plastic material model [8]. In 2001, Yan proposed an equation of residual contact pressure for heat exchanger without considering strain hardening [9].

Wang studied the hydraulic expansion process of bi-metallic composite pipe in accordance with the principles of graphic method [10]. But his prediction doesn't agree with his experimental data reasonably.

Zheng et al proposed effective approaches for hydro-forming process of bi-metallic composite pipe by assuming plane strain condition and elastic-perfectly plastic material model [11]. It derived expressions for predicting hydro-forming pressure and residual stress of the forming process of bi-metallic composite pipe. The predictions of the formulas agree with the test data of Wang's experiment excellently [10,11]. It shows the reliability of the proposed model and formulas impersonally.

Lu's FEM simulation indicated that the there is significant effects of the geometry of drawing cone on the drawing force [7]. Therefore, it is necessary to discuss the effects of the geometry of drawing cone on the drawing force, so as to include such effects and propose a proper approach for the drawing force estimation of bi-metallic composite pipe forming.

In the present article, it aims to propose a modified approach for drawing force estimation of bi-metallic composite pipe forming by combining the results of FEM in addition to the plane strain condition and elastic-perfectly plastic material model.

\section{Stress status in bulging -forming process}

Shown in Fig. 1 is the initial state of the bi-metallic composite pipe system [11]. The inner and outer radii of the liner pipe are $a$ and $b$, respectively, the wall thickness of the liner pipe is $t=b-a$; the inner and outer radii of the base tube are $c$ and $d$, respectively. Before the forming process, $c>b$, i.e., there exists a gap between the liner and base tubes.

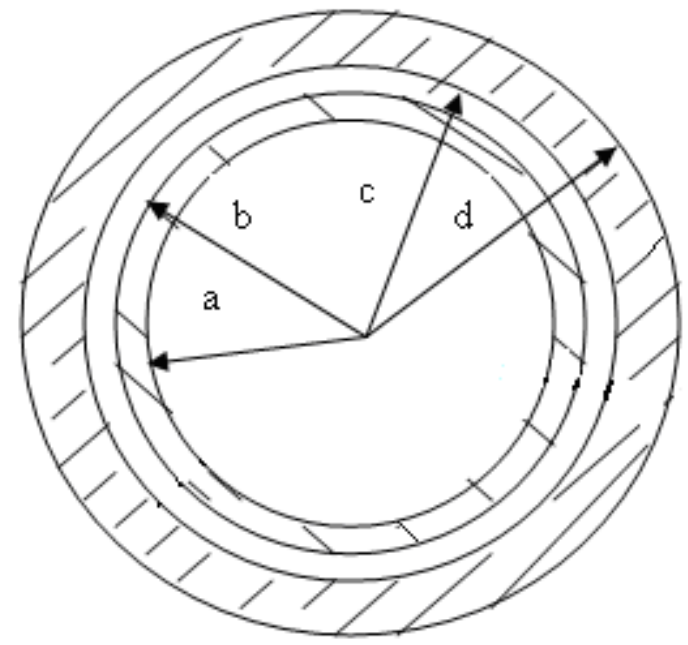

Fig. 1 Schematics of initial state of bi-metallic composite pipe

Fig. 2 shows the stress state. According to the elastic-plastic mechanics principle [11], during the bulging forming process, the liner pipe expands gradually due to the action of the inner pressure, and contacts with the outer tube finally.

Assuming elastic-perfectly plastic material model, under condition of plane strain, the liner pipe deforms plastically and there is no contact pressure until the expanding liner could push the outer base tube to deform.

In the bulging process, it generates contact pressure as long as the liner pipe contacts with the base pipe and could push the base pipe, the base tube withstands the action of the contact pressure. 


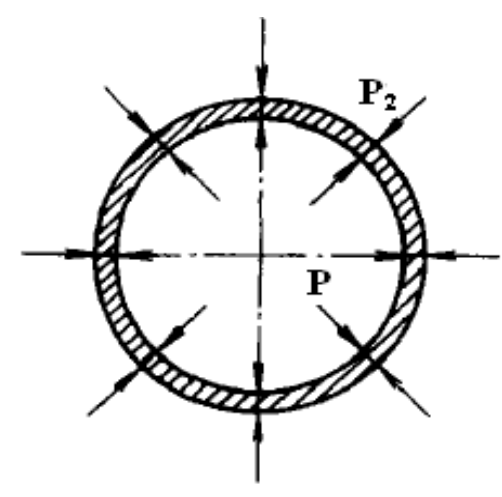

(a)

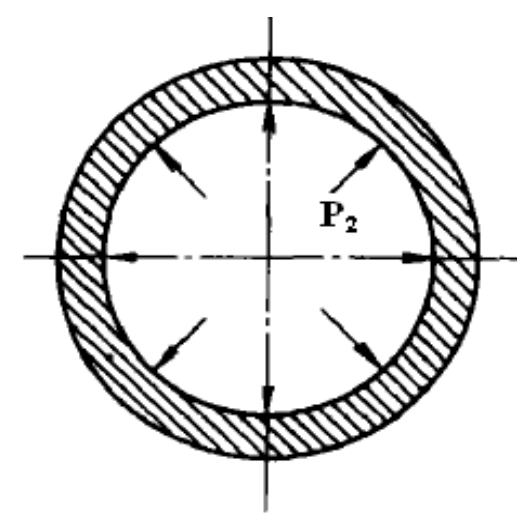

(b)

Fig. 2 Stress status of the bi-metallic composite pipe bulging -forming process

(a) liner; (b) base tube

While, in unloading stage, the internal pressure inside the liner pipe disappears, therefore the liner pipe will shrinks elastically in unloading, the outer edge of the circumference of the liner pipe will shrink as well. So, according to elastic-plastic mechanics principle, the residual pressure at the contact surface in unloading status was obtained and written as [11],

$$
\begin{aligned}
& P= \\
& \frac{2 t \sigma_{s 1}}{b+a}+\left\{P_{r c} \cdot\left\{\frac{1}{E_{1}} \cdot\left[\frac{c^{2}+(c-t \cdot b / c)^{2}}{c^{2}-(c-t \cdot b / c)^{2}}-v_{1}\right]+\frac{1}{E_{2}} \cdot\left[\frac{d^{2}+c^{2}}{d^{2}-c^{2}}+v_{2}\right]\right\}+\frac{\sigma_{s 1}}{E_{1}}\right\} \cdot\left\{E_{2} \cdot\left(1-c^{2} / d^{2}\right) /\left[\left(1-v_{2}\right) c^{2} / d^{2}+\left(1+v_{2}\right)\right]\right\},
\end{aligned}
$$

in which $\sigma_{s 1}$ is the yield strength of the liner material, $P_{r c}$ is the residual pressure at the contact surface after unloading and $P$ is the inner pressure during loading process. $E_{1}$ and $v_{1}$ are elastic modulus and Poisson's ratio of the liner pipe material, $E_{2}$ and $v_{2}$ are elastic modulus and Poisson's ratio of the base pipe material [11].

\section{Drawing force in mechanical drawing process}

Zheng et al analyzed the stress in mechanical drawing process and proposed a simplified expression for the drawing force estimation [11]. As to the manufacture of bi-metallic composite pipe by mechanical drawing method, the main production process is simplified and shown in Fig. 3.

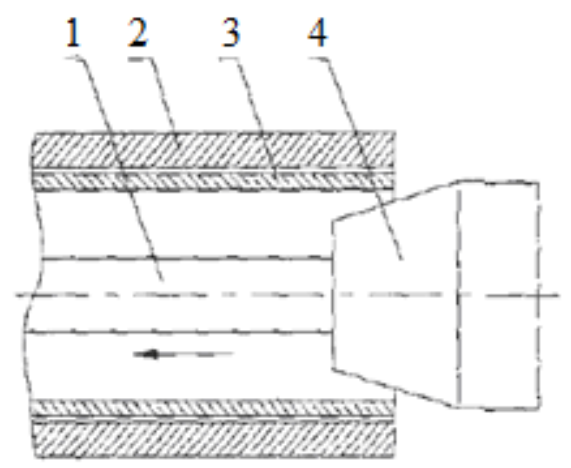

Fig. 3 Manufacture of bi-metallic composite pipe process by drawing method 1-drawbar; 2-base tube; 3-lined pipe; 4-drawing cone

According to Coulomb's friction law, there is a sliding friction coefficient $\mu$ between the cone and the lined pipe as the cone moves during the drawing process. The products of $\mu$ and the inner force of the cone acting the inner line pipe gives the friction force, which is equal to the drawing force $F$ for cone to move forward, thus [11], 


$$
\begin{aligned}
& F=2 \pi c l \mu \cdot P \\
& =2 \pi c l \mu \cdot\left\{P_{r c} \cdot\left\{\frac{1}{E_{1}} \cdot\left[\frac{c^{2}+(c-t \cdot b / c)^{2}}{c^{2}-(c-t \cdot b / c)^{2}}-v_{1}\right]+\frac{1}{E_{2}} \cdot\left[\frac{d^{2}+c^{2}}{d^{2}-c^{2}}+v_{2}\right]\right\} \cdot\left\{\frac{E_{2}\left(1-c^{2} / d^{2}\right)}{\left[\left(1-v_{2}\right) c^{2} / d^{2}+\left(1+v_{2}\right)\right]}\right\} .\right. \\
& +\sigma_{s 1}\left[\frac{2 t}{b+a}+\frac{E_{2}\left(1-c^{2} / d^{2}\right)}{E_{1}\left[\left(1-v_{2}\right) c^{2} / d^{2}+\left(1+v_{2}\right)\right]}\right\}
\end{aligned}
$$

wherein, $l$ is the contact length of the lined pipe and the cone at its maximum cone diameter.

Eq. (2) indicates that the action of the residual pressure at the contact surface $P_{r c}$ and yield strength of the liner material $\sigma_{s 1}$ could be written in twoseparate terms, repectively; the actions of geometric parameters of pipes could be formulated.

The FEM study showed that the there is significant effects of the geometry of drawing cone on drawing force [7]. However, in the above mentioned simplified approach [11], the detailed effects of the geometry of drawing cone on drawing force are not considered. Therefore, it is necessary to deal with the effects of the geometry of drawing cone on drawing force, so as to include such effects and propose a proper approach for drawing force estimation of bi-metallic composite pipe forming.

\section{Modification for drawing force estimation of bi-metallic composite pipe forming}

In reference [7], Lu et al reported their results of FEM simulation for drawing forming process of bi-metallic composite pipe. The lined test tube is $18 \times 1 \mathrm{~mm}$ stainless steel 316 tube, the base tube is $25 \times 3 \mathrm{~mm}$ carbon steel pipe. Thus, the parameters in Fig. 1 are as following, $a=8.0 \mathrm{~mm}, b=9.0 \mathrm{~mm}$, $c=9.5 \mathrm{~mm}, d=12.5 \mathrm{~mm}, t=1.0 \mathrm{~mm}$, and the gap is $1.0 \mathrm{~mm}$. Table 1 shows the parameters of materials properties.

Table 1 Parameters of materials properties

\begin{tabular}{|l|l|l|}
\hline Name & Liner pipe & Base pipe \\
\hline Material Grade & Stainless steel 316 & $20 \#$ \\
\hline Yield strength $\sigma_{\mathrm{s}}(\mathrm{MPa})$ & 255 & 300 \\
\hline Ultimate tensile strength $\sigma_{\mathrm{b}}(\mathrm{MPa})$ & 600 & 485 \\
\hline Elastic modulus E(GPa) & 195 & 206 \\
\hline Poisson's ratio & 0.26 & 0.30 \\
\hline
\end{tabular}

Shown in Fig. 4 is the detail of the drawing process [7]. They considered 6 factors to study the effects of the cone, which includes the semi angle of the cone $\alpha$, the radius of transition circular arc $r$, the length of cone in the maximum diameter part $l$, the sliding friction coefficient $\mu$, the drawing speed $v$, and the diameter of the cone in the maximum diameter part $d$. Orthogonal design was employed to arrange the FEM simulation. 5 levels were selected for each factor. 25 simulations were conducted. The orthogonal design is shown in Table 2 [7].

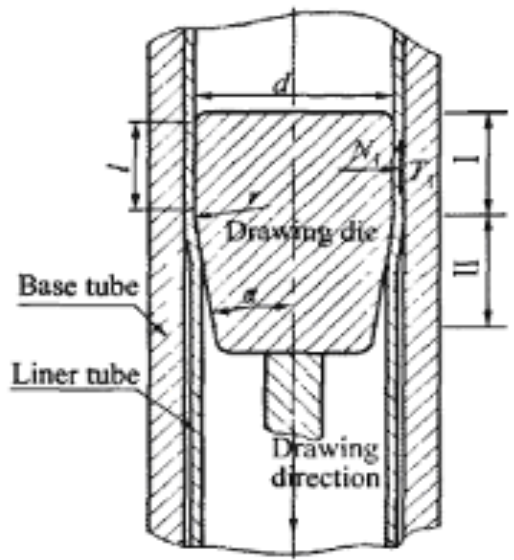

Fig. 4 illustration of drawing forming details 
Table 2 The orthogonal design for the FEM simulation [7]

\begin{tabular}{|c|c|c|c|c|c|c|}
\hline Level No. & $\alpha\left({ }^{9}\right.$ & $r(\mathrm{~mm})$ & $l(\mathrm{~mm})$ & $\mu$ & $v(\mathrm{~m} / \mathrm{s})$ & $d(\mathrm{~mm})$ \\
\hline 1 & 8 & 2 & 6 & 0.05 & 0.01 & 17.1 \\
\hline 2 & 9 & 3 & 7 & 0.10 & 0.02 & 17.2 \\
\hline 3 & 10 & 4 & 8 & 0.15 & 0.03 & 17.3 \\
\hline 4 & 11 & 5 & 9 & 0.20 & 0.04 & 17.4 \\
\hline 5 & 12 & 6 & 10 & 0.25 & 0.05 & 17.5 \\
\hline
\end{tabular}

By substituting the fundamental data of metallic pipes and the property parameters in Table 1 into Eq.(2) of the simple model, we could obtain the following quantitative relationship between the residual stress $P_{r c}$ and the drawing force $F$,

$$
F /(2 \pi l c \mu)=3.4223 P_{r c}+96.7535 \text {. }
$$

On the other hand, we could analyze Lu's FEM data by regression. The result of the regression is shown in Fig.5, the cross symbol " $x$ " indicated the FEM data.

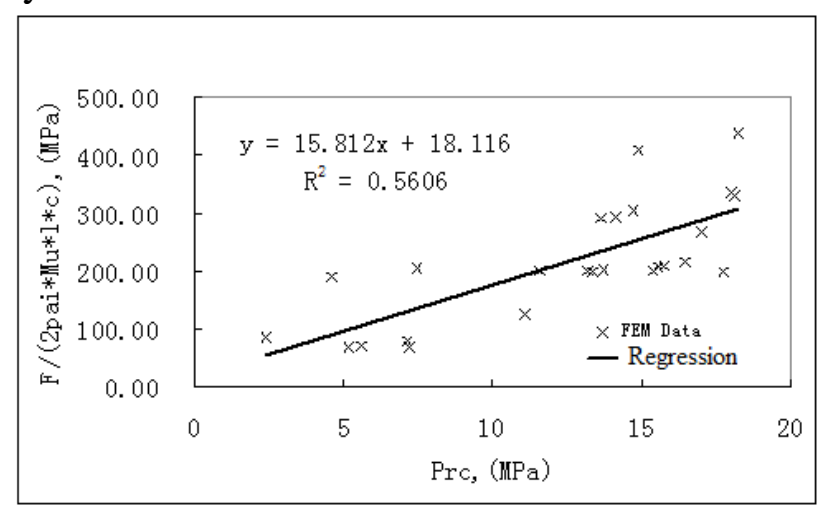

Fig. 5 The results of FEM data and the regression

While, Fig. 5 indicates the relationship between residual stress $P_{r c}$ and the drawing force $F$ is,

$$
F /(2 \pi c \mu)=15.812 P_{r c}+18.116 \text {. }
$$

The correlation factor $R=0.5606^{0.5}=0.7487$, which is bigger than the critical value 0.5052 with the significance of 0.01 for 25 test data.

It is clear that the factors in Eq. (3) and Eq. (4) are different, it indicates the effects of the geometry of drawing cone on drawing force.

Therefore, appropriate factors are needed to introduce in Eq. (3) to include the effects of the geometry of drawing cone on drawing force.

Comparing the results of Eq.(3) and Eq.(4), it derives the factors $\beta$ and $\gamma$ in $P_{r c}$ and $\sigma_{s 1}$ terms, respectively.

The factor in $P_{r c}$ term is,

and the factor in $\sigma_{s 1}$ term is,

$$
\beta=15.812 / 3.4223=4.6203 \text {, }
$$

$$
\gamma=18.116 / 96.7535=0.1872
$$

As a result, the modified drawing force estimation of bi-metallic composite pipe forming could be written as,

$$
\begin{aligned}
& F=2 \pi c l \mu \cdot P \\
& =2 \pi c l \mu \cdot\left\{P_{r c} \cdot \beta \cdot\left\{\frac{1}{E_{1}} \cdot\left[\frac{c^{2}+(c-t \cdot b / c)^{2}}{c^{2}-(c-t \cdot b / c)^{2}}-v_{1}\right]+\frac{1}{E_{2}} \cdot\left[\frac{d^{2}+c^{2}}{d^{2}-c^{2}}+v_{2}\right]\right\} \cdot\left\{\frac{E_{2}\left(1-c^{2} / d^{2}\right)}{\left[\left(1-v_{2}\right) c^{2} / d^{2}+\left(1+v_{2}\right)\right]}\right\},\right. \\
& +\sigma_{s 1} \cdot \gamma \cdot\left[\frac{2 t}{b+a}+\frac{E_{2}\left(1-c^{2} / d^{2}\right)}{E_{1}\left[\left(1-v_{2}\right) c^{2} / d^{2}+\left(1+v_{2}\right)\right]}\right\}
\end{aligned}
$$

i.e., 


$$
\begin{aligned}
& F=2 \pi c l \mu \cdot P \\
& =2 \pi c l \mu \cdot\left\{P_{r c} \cdot 4.6203 \cdot\left\{\frac{1}{E_{1}} \cdot\left[\frac{c^{2}+(c-t \cdot b / c)^{2}}{c^{2}-(c-t \cdot b / c)^{2}}-v_{1}\right]+\frac{1}{E_{2}} \cdot\left[\frac{d^{2}+c^{2}}{d^{2}-c^{2}}+v_{2}\right]\right\} \cdot\left\{\frac{E_{2}\left(1-c^{2} / d^{2}\right)}{\left[\left(1-v_{2}\right) c^{2} / d^{2}+\left(1+v_{2}\right)\right]}\right\}\right. \\
& +\sigma_{s 1} \cdot 0.1872 \cdot\left[\frac{2 t}{b+a}+\frac{E_{2}\left(1-c^{2} / d^{2}\right)}{E_{1}\left[\left(1-v_{2}\right) c^{2} / d^{2}+\left(1+v_{2}\right)\right]}\right\}
\end{aligned}
$$

Eq. (8) is the modified relationship for correlating the drawing for $F$ and the residual stress $P_{r c}$, which includes the detailed effects of the geometry of drawing cone on drawing force.

\section{Conclusion}

In this article, a modified approach for drawing force estimation of bi-metallic composite pipe forming is proposed. The result of FEM is employed to perform the modification, beside the fundamental assumptions of plane strain and elastic-perfectly plastic material model. The modified approach includes the detailed effects of the geometry of drawing cone on drawing force, as well as the properties and geometries of the pipes.

\section{References}

[1] H. Haghighat, G.R. Asgari: International Journal of Mechanical Sciences Vol. 53(2011), p.248.

[2] Marko Knezevic, Mohammad Jahedi, Yannis P. Korkolis, Irene J. Beyerlein: Computational Materials Science Vol. 95(2014), p63.

[3] N.R. Chitkara, A. Aleem: International Journal of Mechanical Sciences Vol. 43(2001), p.2833.

[4] Rimma Lapovok, Hoi Pang Ng, Dacian Tomus, Yuri Estrin: Scripta Materialia Vol. 66(2012), p.1081.

[5] X. Yang, F. Sun, Z. Zhang, H. Shen, S. Guo: The Chinese Journal of Nonferrous Metals Vol. 18 (2008), p.2245.

[6] L. Xue, Y. He, R. Liu, C. Dai, J. Chen: Journal of Plasticity Engineering Vol. 12(2005), p. 74.

[7] X. Lu, X. Zheng: The Chinese J of Nonferrous Metals Vol. 21 (2011), p.205.

[8] Masashi Takemoto: Pressure Vessel Vol. 2(1984), p.68.

[9] H. Yan, C. Yu: Chemical Machinery Vol. 28(2001), p.211.

[10] X. Wang, P. Li, R. Wang: Int. J. of Machine Tools \& Manuf. Vol. 45(2005), p.373.

[11] M. Zheng, H. Gao, H. Teng, et al: Archives of Metallurgy and Materials, Vol. 62 (2017), in press. 